\title{
Erratum to: Numerical modeling of fracking fluid migration through fault zones and fractures in the North German Basin
}

Helena Pfunt ${ }^{1}$ - Georg Houben ${ }^{1}$. Thomas Himmelsbach ${ }^{1}$

Published online: 23 August 2016

(C) Springer-Verlag Berlin Heidelberg 2016

Erratum to: Hydrogeol J (2016)

DOI 10.1007/s10040-016-1418-7

In equation (3), $v$ was incorrectly defined. It should be kinematic viscosity, thus:

$K=\frac{g}{12 v} b^{2}$

with:

$K$ - hydraulic conductivity $(\mathrm{m} / \mathrm{s})$

$g-$ gravitational acceleration $\left(\mathrm{m} / \mathrm{s}^{2}\right)$

$v$ - kinematic viscosity $\left(\mathrm{m}^{2} / \mathrm{s}\right)$

$b-$ fracture aperture $(\mathrm{m})$

The online version of the original article can be found at http://dx.doi. org/10.1007/s10040-016-1418-7.

Helena Pfunt

helena.pfunt@bgr.de

Federal Institute for Geosciences and Natural Resources (BGR),

Stilleweg 2, 30655 Hanover, Germany 